\title{
Nonlinear dynamics of a submerged floating moored structure by incremental harmonic balance method with FFT
}

\author{
Wei Lu, Fei Ge*, Xiaodong Wu, Youshi Hong \\ State Key Laboratory of Nonlinear Mechanics, Institute of Mechanics, Chinese Academy of Sciences, \\ No.15 Beisihuanxi Road, Beijing 100190, China
}

\section{A R T I C L E I N F}

\section{Article history:}

Received 20 July 2011

Received in revised form 6 September 2012

Accepted 4 January 2013

\section{Keywords:}

Submerged floating moored structure

Nonlinear dynamics

Incremental harmonic balance

Chaotic motion

Period doubling bifurcation

Domains of attraction

\begin{abstract}
A B S T R A C T
A submerged floating moored structure has a great potential in ocean engineering applications. The nonlinear dynamics of a submerged floating moored structure subjected to vertical excitation with possible slackness in the mooing system are investigated by incremental harmonic balance (IHB) method. Heaviside step function is introduced to describe the nonlinearity in axial stiffness arising from loss of mooring tension. The dimensionless governing equation is derived, and three parameters, frequency ratio $\eta$, damping ratio $\zeta$ and dimensionless net buoyancy $W$, are found to be independent. Due to the fact that the restoring force term is function of the unknown displacement and could barely be expressed in an explicit form of time, a fast Fourier transformation (FFT) is implemented in IHB method to simplify the Galerkin average procedure. Both stable and unstable solutions and both period- 1 and bifurcated solutions are obtained by IHB method. The stability of the periodic solutions is investigated by Floquet theory. Parameter study is carried out. Results indicate that the system nonlinearity becomes stronger as dimensionless the net buoyancy $W$ and damping ratio $\zeta$ decrease. A path to chaotic motions though a series of period doubling bifurcations is found. Multiple solutions are observed, and the domains of attraction are investigated by interpolated cell mapping (ICM) technique.
\end{abstract}

() 2013 Elsevier Ltd. All rights reserved.

\footnotetext{
* Corresponding author.

E-mail address: gefei@imech.ac.cn (F. Ge).
} 


\section{Introduction}

A submerged moored floating structure has great potential in ocean engineering applications, such as breakwaters [1-3] or submerged floating tunnels (SFT) [4,5], etc. It provides an alternative for the cases with poor foundation or large water depth that exclude the possibility of fixed bottom-founded structures, or the cases with a requirement of a clear water surface. With the crest submerged under water, it not only allows water surface activities but also avoids generating significant reflected wave and affecting the nearby structures compared to emerging structures. Besides, it is more favorable from a viewpoint of economics with relative low expenses, and also from a viewpoint of environment protection with allowance for the free exchange of sea water comparing to fixed structures.

The mooring system provides a restrain to the submerged floating moored structure to keep it in position by tether tension. Generally a large initial mooring tension is guaranteed to provide an effective restrain to the structure in service; while on the other hand, the increase in the static initial mooring tension would be costly as it lays more strict safety requirements on the mooring components. However, despite a large static initial mooring tension, in an actual sea environment the occurrence of occasional loss of tension is sometimes inevitable when confronting a rough environment conditions such as severe sea waves or seismic activities. The loss of mooring tension will introduce a strong nonlinearity in the mechanical property of mooring system, which is the discontinuity in the tether axial stiffness, as the mooring components are generally slender structures and can barely resist compression. Due to the remarkable nonlinear property of the mooring system, some nonlinear dynamic phenomena of the cable-body system operating in an alternate slack taut state, such as the socalled 'snap force', which is the impact tension load in the tether, are reported in several experimental and numerical researches.

Generally the numerical simulation of this type of problem is based on time domain due to the nonlinear nature. Liu [6] used a two dimensional model to compute snap loads in lifting and mooring lines. Niedzwecki and Thampi [7] proposed a two-part analysis procedure to predict snap loads behavior of marine cable-body systems. Huang and Vassalos [8] developed a three-dimensional model based on lumped-mass-and-spring method to predict the dynamics of marine cables operating in alternating taut-slack conditions. Patel and Park $[9,10]$ presented an extension of the theory of pulse buckling for the dynamics of TLP tethers at low tension. Vassalos and Huang [11] studied the dynamics of small-sagged taut-slack marine cables by Galerkin method. Plaut et al. [12] investigated the threedimensional dynamics of a moored cylinder used as a breakwaters with considering the snap loading in the mooring lines. Lu et al. [13] investigated the slack phenomena in tethers of submerged floating tunnels (SFT) and proposed a procedure for preliminary slack prediction. Vassalos et al. [14] carried out a series of model experiments to investigate the dynamics of a marine cables with the occurrence of slacking. Kunisu et al. [15] investigated the dynamics of a submerged floating tunnel in wave tank, and analyzed the snap force in the mooring tether.

The semi-analytical method-incremental harmonic balance (IHB) method is used to investigate the nonlinear dynamics of submerged floating moored structures with possible slackness in the mooing system. IHB method is a powerful approach recently developed for nonlinear analysis with a great advantage in dealing with strong nonlinearities comparing to the classic approaches. Moreover it is remarkably effective in computer implementation for obtaining response with desired accuracy over a wide range of varying parameter with both stable and unstable solutions. By iteration starting from the solutions obtained from an adjacent parameter value, a new solution will converge fastly, which indicates that IHB method is an ideal approach to perform parametric study with high efficiency. The method was originally proposed by Cheung and Lau [16,17]. Cheung et al. [18] then applied the method to cubic nonlinearity systems, and adopted an incremental arc-length method with a cubic extrapolation technique to trace the response curve. Lau and Zhang [19] extended the method to analyze the nonlinear vibrations of piecewise-linear system, with the idea then widely used in the piecewise-linear system [20,21]. Leung and Chui [22] applied the method to Duffing oscillators.

In present research, the nonlinear dynamics of a submerged floating moored structure subjected to vertical excitation with the possible slackness in the mooing system is considered. The Heaviside step function is introduced to describe the nonlinearity in the axial stiffness arising from the loss of mooring tension. As the restoring force term could hardly be expressed explicitly as the function of time, IHB 
method with an implement of FFT is used. The non-dimensional governing equation and its IHB formulation are derived. The stability of the periodic solutions is investigated by Floquet theory, and the transition matrix is calculated by the method in Ref. [23]. Parametric study is carried out, and multiple solutions are found at certain conditions. The domains of attraction are obtained by interpolated cell mappings (ICM) method [24].

\section{Governing equation}

As depicted in Fig. 1, a submerged vertical moored floating structure subjected to a vertical sinusoidal excitation is considered. The mass of the floating structure is $m$ (including added mass), and the net buoyancy is $w$. A space fixed coordinate is built with its origin point $o$ located at the un-stretched end of the mooring tether, and the ox axis is parallel to the vertical line. The tensile stiffness of the tether is $k_{0}$, while when the motion of the floating structure goes large and the mooring tether become slack, it will reduce to zero. Therefore the Heaviside step function is introduced to describe the restoring force term. An linearized fluid drag force model are adopted to calculate the fluid drag force on the submerged moored floating structure in present research, the motion of the submerged moored floating structure can be expressed as:

$$
m \ddot{x}+c \dot{x}+H(x) k_{0} x=w+f_{0} \sin \omega_{f} t
$$

in which $c$ is the drag coefficient of the fluid force on the submerged moored floating structure; $f_{0}$ and $\omega_{f}$ are the amplitude and circular frequency of the external excitation respectively; the dots over the variables denote differentiation with respect to $t H(x)$ is the Heaviside step function:

$$
H(x)= \begin{cases}1 & x \geq 0 \\ 0 & x<0\end{cases}
$$

The system will degenerate into linear system when the motion of the structure is small and $H(x)$ remains to be constant 1 .

By introducing a dimensionless time scale $\tau=\omega_{f} t$, natural frequency $\omega_{n}=\sqrt{k_{0} / m}$, frequency ratio $\eta=\omega_{f} / \omega_{n}$, and damping ratio $\zeta=c /\left(2 m \omega_{n}\right)$, the governing equation of the system Eq. (1) is transformed into:

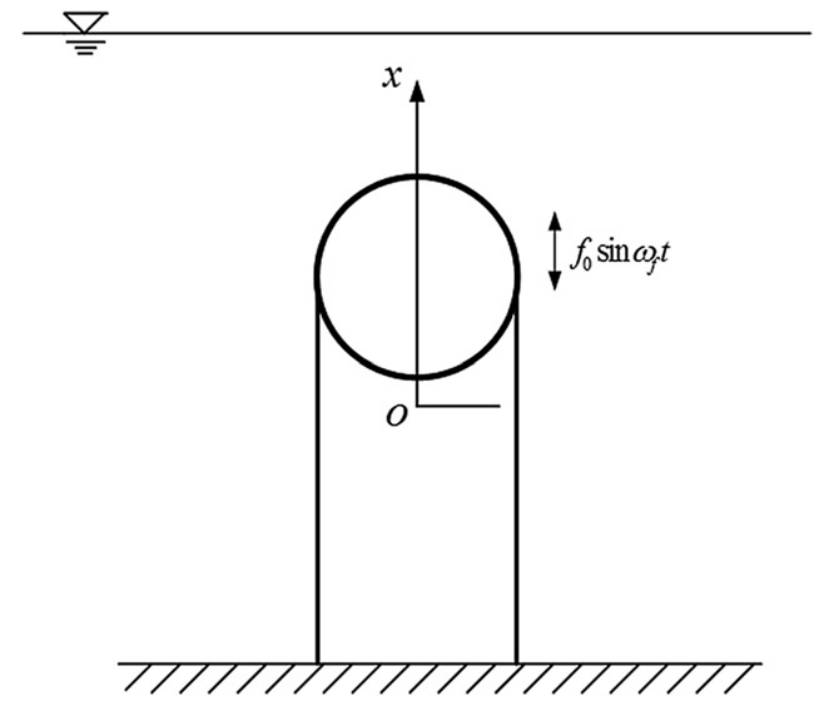

Fig. 1. A submerged vertical moored floating structure. 


$$
\eta^{2} x^{\prime \prime}+2 \zeta \eta x^{\prime}+H(x) x=\frac{w}{m \omega_{n}^{2}}+\frac{f_{0}}{m \omega_{n}^{2}} \sin \tau
$$

in which $x^{\prime}=d x / d \tau, x^{\prime \prime}=d^{2} x / d \tau^{2}$.

Defining a static displacement $x_{0}=f_{0} / m \omega_{n}^{2}=f_{0} / k_{0}$, and then introducing the dimensionless displacement $X=x / x_{0}$ and non-dimensional net buoyancy $W=w / f_{0}$, which is the ratio of net buoyancy and amplitude of external excitation, we obtain:

$$
\begin{aligned}
& \eta^{2} X^{\prime \prime}+2 \zeta \eta X^{\prime}+G(X)=\sin \tau \\
& G(X)=H(X) X-W
\end{aligned}
$$

The non-dimensional governing equation (3) indicates that for the present system only three parameters are independent, that is frequency ratio $\eta$, damping ratio $\zeta$ and dimensionless net buoyancy $W$; while the amplitude of external excitation force $f_{0}$ does not appear as an independent parameter. The influence of amplitude of external excitation is reflected by the dimensionless net buoyancy $W$ and the amplification of dimensionless displacement $X$.

\section{IHB method with FFT}

For a nonlinear system in the general form of:

$$
f(\ddot{x}, \dot{x}, x, t)=0
$$

by introducing an increment around the exact solution $x_{0}: x=x_{0}+\Delta x$, the incremental formation of the original equation can be obtained as:

$$
f\left(\ddot{x}_{0}+\Delta \ddot{x}, \dot{x}_{0}+\Delta \dot{x}, x_{0}+\Delta x, t\right)=0
$$

expanding equation (6) using Taylor series, we get:

$$
f\left(\ddot{x}_{0}, \dot{x}_{0}, x_{0}, t\right)+\left.\frac{\partial f}{\partial \ddot{x}}\right|_{\ddot{x}_{0}} \Delta \ddot{x}+\left.\frac{\partial f}{\partial \dot{x}}\right|_{\dot{x}_{0}} \Delta \dot{x}+\left.\frac{\partial f}{\partial x}\right|_{x_{0}} \Delta x+\text { Higher }- \text { order terms }=0
$$

here $R\left(x_{0}\right)=-f\left(\ddot{x}_{0}, \dot{x}_{0}, x_{0}, t\right)$ is defined as the residual term, which is zero when is the exact solution. Neglecting the higher order terms, the incremental form of the original equation (6) is then obtained:

$$
\left.\frac{\partial f}{\partial \ddot{x}}\right|_{\ddot{x}_{0}} \Delta \ddot{x}+\left.\frac{\partial f}{\partial \dot{x}}\right|_{\dot{x}_{0}} \Delta \dot{x}+\left.\frac{\partial f}{\partial x}\right|_{x_{0}} \Delta x=R\left(x_{0}\right)
$$

it is assumed that the solution $x$ is in the Fourier series form and its increment is $\Delta x$ :

$$
\begin{aligned}
& x(t)=a_{0}+\sum_{i=1}^{N H}\left(a_{i} \cos i t+b_{i} \sin i t\right) \\
& \Delta x(t)=\Delta a_{0}+\sum_{i=1}^{N H}\left(\Delta a_{i} \cos i t+\Delta b_{i} \sin i t\right)
\end{aligned}
$$

in which, $N H$ is the number of the harmonic components in the Fourier series, $a_{i}, b_{i}, \Delta a_{i}, \Delta b_{i}$ are the corresponding Fourier coefficients of $x(t)$ and $\delta(\Delta x)$ respectively.

Substitute the Fourier expression (9) and (10) of $x$ and $\Delta x$ into the incremental differential equation (8), it can be transformed into a group of algebraic equations about the incremental coefficients $\Delta a_{i}, \Delta b_{i}$ by applying Galerkin average procedure: 


$$
\int_{0}^{2 \pi}\left(\left.\frac{\partial f}{\partial \ddot{x}}\right|_{\ddot{x}_{0}} \Delta \dot{x}+\left.\frac{\partial f}{\partial \dot{x}}\right|_{\dot{x}_{0}} \Delta \dot{x}+\left.\frac{\partial f}{\partial x}\right|_{x_{0}} \Delta x\right) \cdot \delta(\Delta x) d t=\int_{0}^{2 \pi} R\left(x_{0}\right) \cdot \delta(\Delta x) d t
$$

in which $\delta(\Delta x)$ is variation of $\Delta x$. Expand $\delta(\Delta x)$ of equation (11) with its Fourier form as in (10)

$$
\begin{aligned}
& \sum_{i=0}^{N H}\left[\int_{0}^{2 \pi}\left(\left.\frac{\partial f}{\partial \ddot{x}}\right|_{\ddot{x}_{0}} \Delta \ddot{x}+\left.\frac{\partial f}{\partial \dot{x}}\right|_{\dot{x}_{0}} \Delta \dot{x}+\left.\frac{\partial f}{\partial x}\right|_{\chi_{0}} \Delta x\right) \cos i t \cdot d t \cdot \delta\left(\Delta a_{i}\right)+\int_{0}^{2 \pi}\left(\left.\frac{\partial f}{\partial \ddot{x}}\right|_{\ddot{x}_{0}} \Delta \ddot{x}+\left.\frac{\partial f}{\partial \dot{x}}\right|_{\dot{x}_{0}} \Delta \dot{x}+\left.\frac{\partial f}{\partial x}\right|_{x_{0}} \Delta x\right)\right. \\
& \left.\sin i t \cdot d t \cdot \delta\left(\Delta b_{i}\right)\right]=\sum_{i=0}^{N H}\left[\int_{0}^{2 \pi} R\left(x_{0}\right) \cos i t \cdot d t \cdot \delta\left(\Delta a_{i}\right)+\int_{0}^{2 \pi} R\left(x_{0}\right) \sin i t \cdot d t \cdot \delta\left(\Delta b_{i}\right)\right]
\end{aligned}
$$

Let the coefficients of the corresponding variation terms $\delta\left(\Delta a_{i}\right), \delta\left(\Delta b_{i}\right)$ of both sides of the equation equal, a group of algebraic equations can be obtained

$$
[\mathbf{C}]\{\Delta \mathbf{q}\}=\{\mathbf{R}\}
$$

where $[\mathbf{C}]$ is the Jacobian matrix, $\{\Delta \mathbf{q}\}$ is the vector of incremental coefficients and $\{\mathbf{R}\}$ is residual vector.

While in fact, the coefficient functions $\partial f /\left.\partial \ddot{x}\right|_{\dot{x}_{0}}$, $\partial f /\left.\partial \dot{x}\right|_{\dot{x}_{0}} \partial f /\left.\partial x\right|_{x_{0}}$, of the incremental differential equation (8) are sometimes hardly expressed in explicit forms of time. In the present system, the restoring force term $G(X)$ is the function of displacement $X$, which is difficult to be expressed as the function of time explicitly with $X$ unresolved. Therefore, IHB method with an implement of FFT is used to simplify the Galerkin average procedure, which has been proved to be of great efficiency. The coefficient functions are denoted as:

$$
\left.\frac{\partial f}{\partial \ddot{x}}\right|_{\ddot{x}_{0}}=\varphi(t),\left.\frac{\partial f}{\partial \dot{x}}\right|_{\dot{x}_{0}}=u(t),\left.\frac{\partial f}{\partial x}\right|_{x_{0}}=k(t), R\left(x_{0}(t)\right)=r(t)
$$

then applying FFT to these functions using \{cosit,sinit $\}$ as the bases, we get:

$$
\left\{\begin{array}{l}
\varphi(t) \\
u(t) \\
k(t) \\
r(t)
\end{array}\right\}=\left\{\begin{array}{l}
\varphi_{0} \\
u_{0} \\
k_{0} \\
r_{0}
\end{array}\right\}+\sum_{i=1}^{N F F T}\left(\left\{\begin{array}{l}
\varphi_{i}^{c} \\
u_{i}^{c} \\
k_{i}^{c} \\
r_{i}^{c}
\end{array}\right\} \cos i t+\left\{\begin{array}{c}
\varphi_{i}^{s} \\
u_{i}^{s} \\
k_{i}^{s} \\
r_{i}^{s}
\end{array}\right\} \sin i t\right)
$$

where $N F F T \geq 2 N H$.

With the coefficient functions in Fourier form, the incremental differential equation can be expressed as:

$$
\begin{aligned}
& {\left[\varphi_{0}+\sum_{i=1}^{N F F T}\left(\varphi_{i}^{c} \cos i t+\varphi_{i}^{S} \sin i t\right)\right] \Delta \ddot{x}+\left[u_{0}+\sum_{i=1}^{N F F T}\left(u_{i}^{c} \cos i t+u_{i}^{S} \sin i t\right)\right]} \\
& +\Delta \dot{x}+\left[k_{0}+\sum_{i=1}^{N F F T}\left(k_{i}^{c} \cos i t+k_{i}^{S} \sin i t\right)\right] \Delta x \quad=r_{0}+\sum_{i=1}^{N F F T}\left(r_{i}^{c} \cos i t+r_{i}^{S} \sin i t\right)
\end{aligned}
$$

Substitute the Fourier form of $\Delta x$ as in expression (10) and its differentials $\Delta \dot{x}, \Delta \ddot{x}$ into equation (16)

$$
\begin{aligned}
& {\left[\varphi_{0}+\sum_{i=1}^{N F F T}\left(\varphi_{i}^{c} C_{i}+\varphi_{i}^{s} S_{i}\right)\right]\left[\sum_{j=1}^{N H}\left(-\Delta a_{j} j^{2} C_{j}-\Delta b_{j} j^{2} S_{j}\right)\right]+\left[u_{0}+\sum_{i=1}^{N F F T}\left(u_{i}^{c} C_{i}+u_{i}^{s} S_{i}\right)\right]} \\
& {\left[\sum_{j=1}^{N H}\left(-\Delta a_{j} S_{j}+\Delta b_{j} C_{j}\right)\right]+\left[k_{0}+\sum_{i=1}^{N F F T}\left(k_{i}^{c} C_{i}+k_{i}^{S} S_{i}\right)\right]\left[a_{0}+\sum_{j=1}^{N H}\left(\Delta a_{j} C_{j}+\Delta b_{j} S_{j}\right)\right]} \\
& =r_{0}+\sum_{i=1}^{N F F T}\left(r_{i}^{c} C_{i}+r_{i}^{s} S_{i}\right)
\end{aligned}
$$


where $C_{i}=$ cosit, $S_{i}=$ sinit.

Apply Galerkin average to both sides of equation (17)

$$
\begin{aligned}
& \int_{0}^{2 \pi}\left\{\left[\varphi_{0}+\sum_{i=1}^{N F F T}\left(\varphi_{i}^{c} C_{i}+\varphi_{i}^{S} S_{i}\right)\right]\left[\sum_{j=1}^{N H}\left(-\Delta a_{j} j^{2} C_{j}-\Delta b_{j} j^{2} S_{j}\right)\right]+\left[u_{0}+\sum_{i=1}^{N F F T}\left(u_{i}^{c} C_{i}+u_{i}^{S} S_{i}\right)\right]\right. \\
& {\left.\left[\sum_{j=1}^{N H}\left(-\Delta a_{j} j S_{j}+\Delta b_{j} C_{j}\right)\right]+\left[k_{0}+\sum_{i=1}^{N F F T}\left(k_{i}^{c} C_{i}+k_{i}^{S} S_{i}\right)\right]\left[a_{0}+\sum_{j=1}^{N H}\left(\Delta a_{j} C_{j}+\Delta b_{j} S_{j}\right)\right]\right\} \cdot \delta(\Delta x) d t } \\
&= \int_{0}^{2 \pi}\left[r_{0}+\sum_{i=1}^{N F F T}\left(r_{i}^{c} C_{i}+r_{i}^{S} S_{i}\right)\right] \cdot \delta(\Delta x) d t
\end{aligned}
$$

Expand the variation of $\delta(\Delta x)$ with its Fourier form as in expression (10)

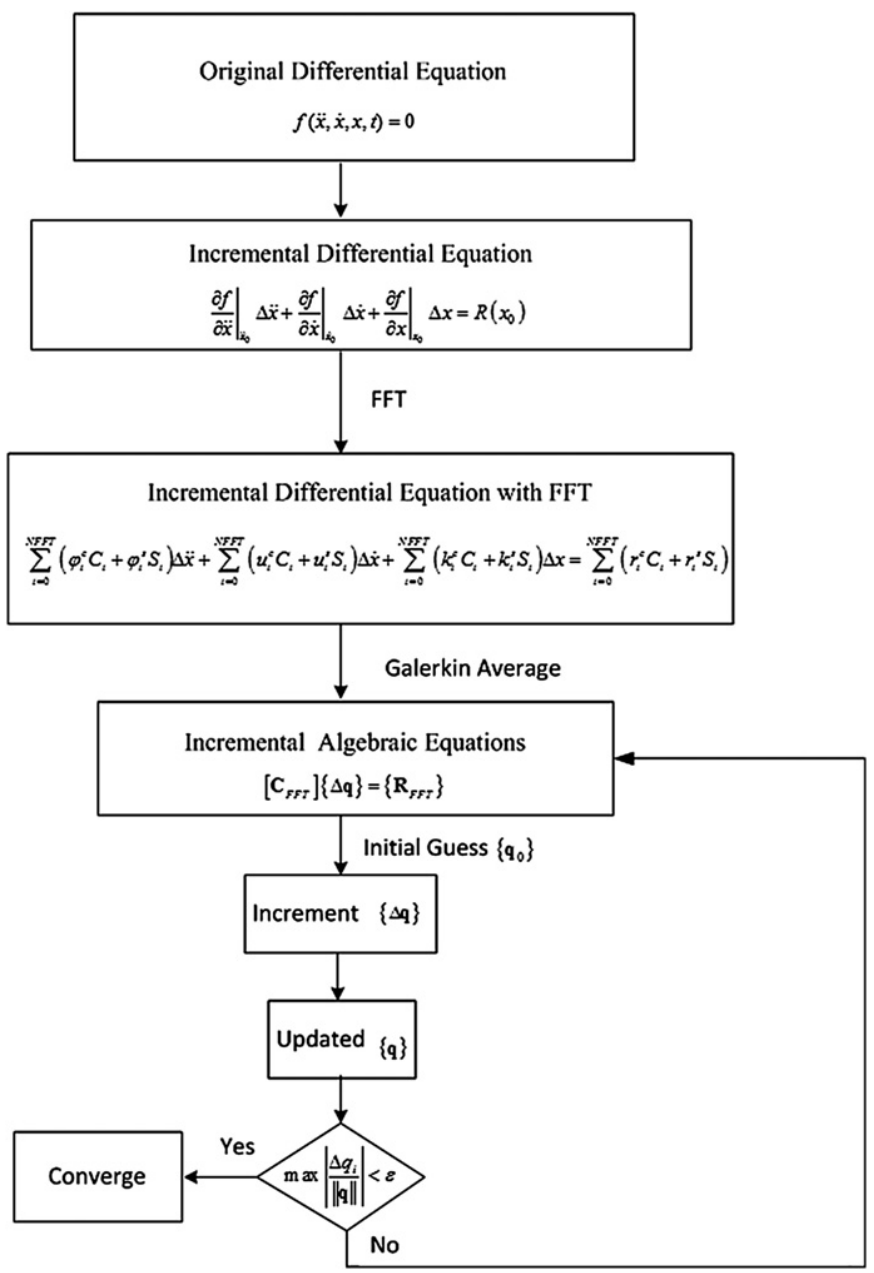

Fig. 2. Flow chart of solving procedure of IHB method with FFT. 
Table 1

Maximum residual values at different harmonic terms $N H$ (period-1 solution).

\begin{tabular}{lllll}
\hline$\eta$ & \multicolumn{2}{l}{$R_{\max }$} & & $N H=40$ \\
\cline { 2 - 5 } & $N H=5$ & $N H=10$ & $N H=20$ & 0.00168 \\
\hline 0.1 & 0.00854 & 0.00796 & 0.00316 & 0.00773 \\
0.5 & 0.06225 & 0.03374 & 0.01602 & 0.00956 \\
1 & 0.08245 & 0.0382 & 0.01977 & $6.66134 \mathrm{E}-16$ \\
1.5 & $4.44089 \mathrm{E}-16$ & $4.44089 \mathrm{E}-16$ & $5.55112 \mathrm{E}-16$ & $4.44089 \mathrm{E}-16$ \\
2 & $3.33067 \mathrm{E}-16$ & $3.33067 \mathrm{E}-16$ & $4.44089 \mathrm{E}-16$ & $4.44089 \mathrm{E}-16$ \\
2.5 & $3.33067 \mathrm{E}-16$ & $3.33067 \mathrm{E}-16$ & $4.44089 \mathrm{E}-16$ & $5.55112 \mathrm{E}-16$ \\
3 & $4.44089 \mathrm{E}-16$ & $4.44089 \mathrm{E}-16$ & $5.55112 \mathrm{E}-16$ & \\
\hline
\end{tabular}

$$
\begin{aligned}
& \int_{0}^{2 \pi}\left\{\left[\varphi_{0}+\sum_{i=1}^{N F F T}\left(\varphi_{i}^{c} C_{i}+\varphi_{i}^{S} S_{i}\right)\right]\left[\sum_{j=1}^{N H}\left(-\Delta a_{j} j^{2} C_{j}-\Delta b_{j} j^{2} S_{j}\right)\right]+\left[u_{0}+\sum_{i=1}^{N F F T}\left(u_{i}^{c} C_{i}+u_{i}^{s} S_{i}\right)\right]\right. \\
& {\left.\left[\sum_{j=1}^{N H}\left(-\Delta a_{j} j S_{j}+\Delta b_{j} j C_{j}\right)\right]+\left[k_{0}+\sum_{i=1}^{N F F T}\left(k_{i}^{c} C_{i}+k_{i}^{s} S_{i}\right)\right]\left[a_{0}+\sum_{j=1}^{N H}\left(\Delta a_{j} C_{j}+\Delta b_{j} S_{j}\right)\right]\right\} } \\
& \sum_{k=0}^{N H}\left[\delta\left(\Delta a_{k}\right) C_{k}+\delta\left(\Delta b_{k}\right) S_{k}\right] d t=\int_{0}^{2 \pi}\left[r_{0}+\sum_{i=1}^{N F F T}\left(r_{i}^{c} C_{i}+r_{i}^{s} S_{i}\right)\right] \cdot \sum_{k=0}^{N H}\left[\delta\left(\Delta a_{k}\right) C_{k}+\delta\left(\Delta b_{k}\right) S_{k}\right] d t
\end{aligned}
$$
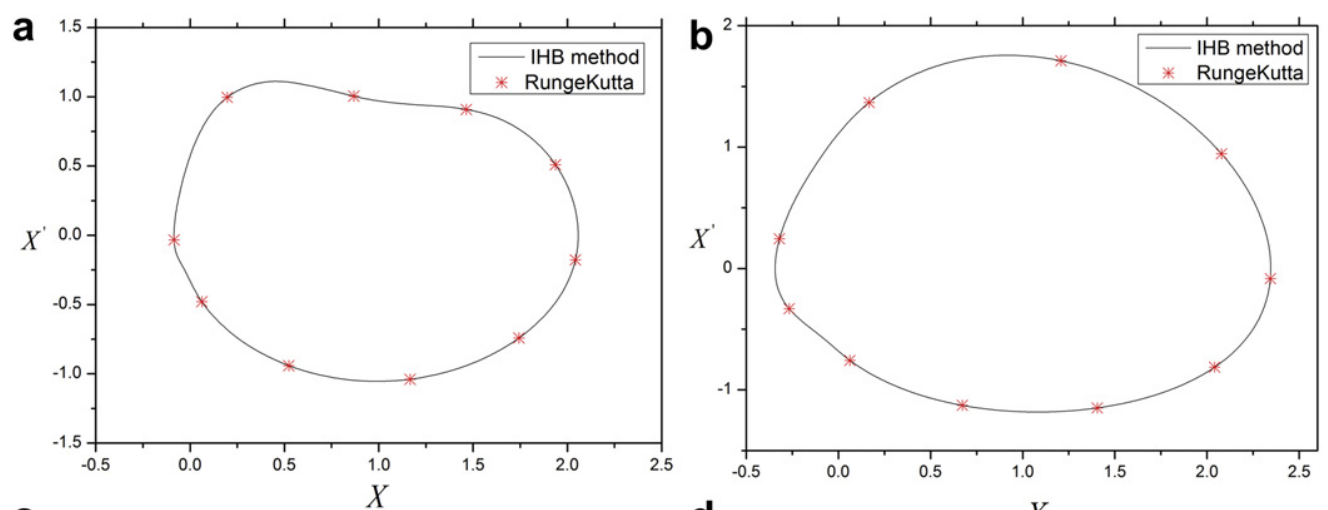

C

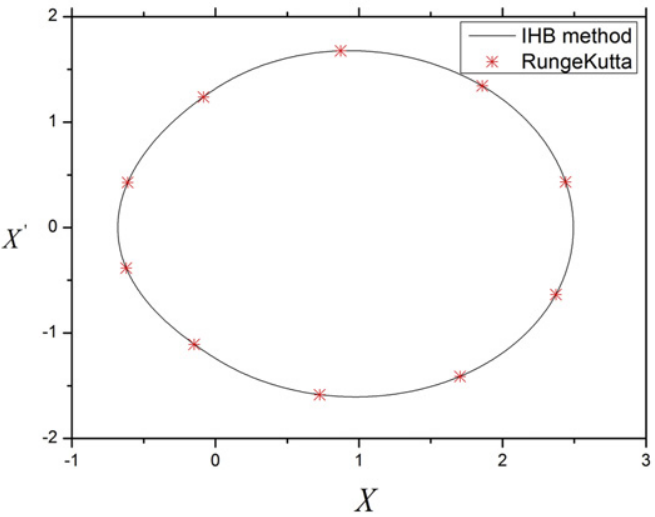

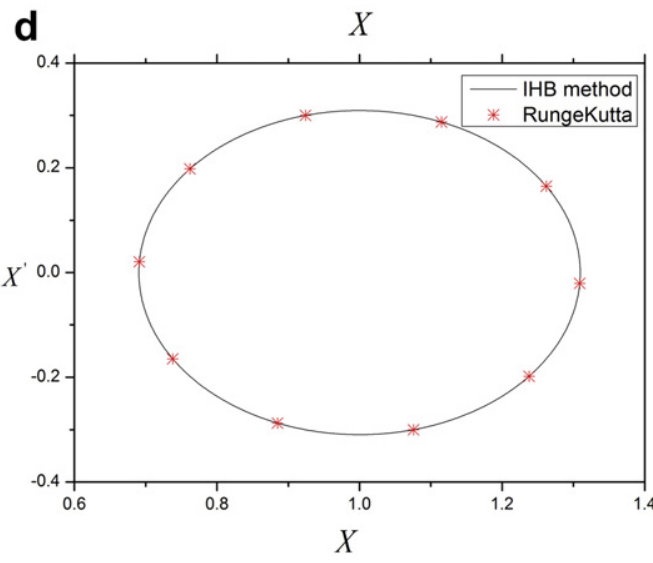

Fig. 3. Phase plane diagrams obtained by IHB method and numerical integration, $W=1, \zeta=0.3$. a. $\eta=0.3$, b. $\eta=0.5$, c. $\eta=1$, d. $\eta=2$. 
The coefficients of corresponding variation terms $\delta\left(\Delta a_{k}\right) \delta\left(\Delta b_{k}\right)$ on the both sides of the equation should be equal, so the following equations can be obtained

$$
\begin{aligned}
\int_{0}^{2 \pi}\left\{\left[\varphi_{0}+\sum_{i=1}^{N F F T}\left(\varphi_{i}^{c} C_{i}+\varphi_{i}^{s} S_{i}\right)\right]\left[\sum_{j=1}^{N H}\left(-\Delta a_{j} j^{2} C_{j}-\Delta b_{j} j^{2} S_{j}\right)\right]+\left[u_{0}+\sum_{i=1}^{N F F T}\left(u_{i}^{c} C_{i}+u_{i}^{s} S_{i}\right)\right]\right. \\
\left.\quad\left[\sum_{j=1}^{N H}\left(-\Delta a_{j} j S_{j}+\Delta b_{j} j C_{j}\right)\right]+\left[k_{0}+\sum_{i=1}^{N F F T}\left(k_{i}^{c} C_{i}+k_{i}^{s} S_{i}\right)\right]\left[a_{0}+\sum_{j=1}^{N H}\left(\Delta a_{j} C_{j}+\Delta b_{j} S_{j}\right)\right]\right\} C_{k} d t \\
\int_{0}^{2 \pi}\left\{\left[r_{0}+\sum_{i=1}^{N F F T}\left(r_{i}^{c} C_{i}+r_{i}^{s} S_{i}\right)\right] C_{k} \cdot d t(k=0,1,2 \ldots N H)\right. \\
{\left[\sum_{i=1}^{N H}\left(\varphi_{i}^{c} C_{i}+\varphi_{i}^{s} S_{i}\right)\right]\left[\sum_{j=1}^{N H}\left(-\Delta a_{j} j^{2} C_{j}-\Delta b_{j} j^{2} S_{j}\right)\right]+\left[u_{0}+\sum_{i=1}^{N F F T}\left(u_{i}^{c} C_{i}+u_{i}^{s} S_{i}\right)\right] } \\
\left.=\int_{j=1}^{2 \pi}\left[r_{0}+\sum_{i=1}^{N F F T}\left(r_{i}^{c} C_{i}+\Delta b_{j} j C_{j}\right)\right]+\left[r_{i}^{S} S_{i}\right)\right] S_{k} \cdot d t(k=1,2 \ldots, N H) \\
\end{aligned}
$$

then the incremental algebraic equations can be derived from (20) and (21):

$$
\left[\mathbf{C}_{\mathrm{FFT}}\right]\{\Delta \mathbf{q}\}=\left\{\mathbf{R}_{\mathrm{FFT}}\right\}
$$

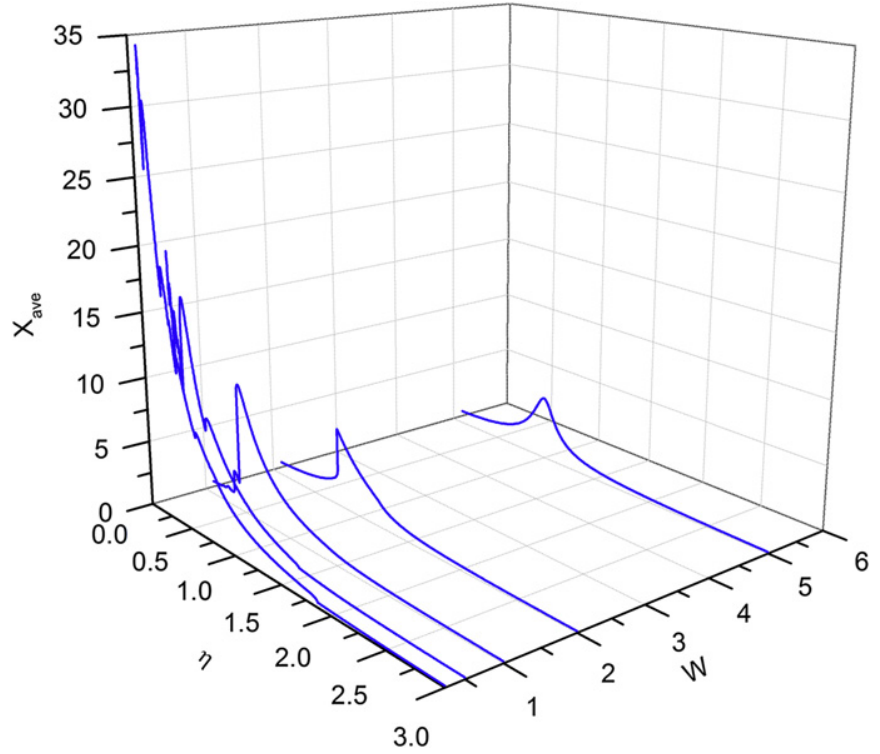

Fig. 4. Three-dimensional view of frequency-response curves under different dimensionless net buoyancy. 
in which

$$
\begin{aligned}
& {\left[C_{\mathrm{FFT}}\right]=\left[\begin{array}{lll}
C_{00} & A_{0 j} & B_{0 j} \\
C_{i 0}^{C} & A_{i j}^{C} & B_{i j}^{C} \\
C_{i 0}^{S} & A_{i j}^{S} & A_{i j}^{S}
\end{array}\right] i, j=1,2, \ldots N H} \\
& \Delta \mathbf{q}=\left\{\Delta a_{0}, \Delta a_{1}, \ldots, \Delta a_{N H}, \Delta b_{1}, \ldots, \Delta b_{N H}\right\}^{T} \\
& \left\{\mathbf{R}_{\mathrm{FFT}}\right\}=\left\{R_{0}, \mathbf{R}^{C}, \mathbf{R}^{S}\right\}
\end{aligned}
$$

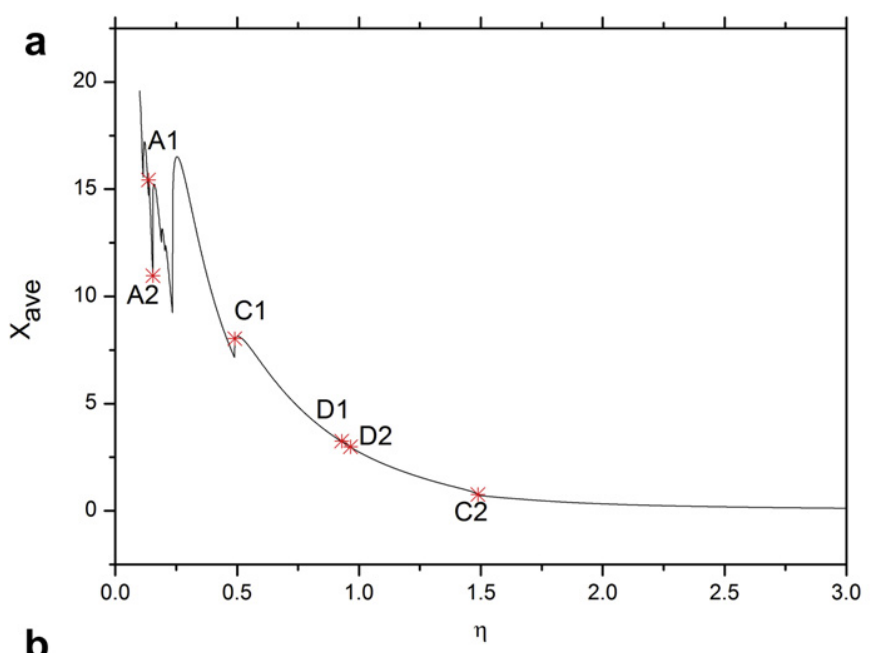

b

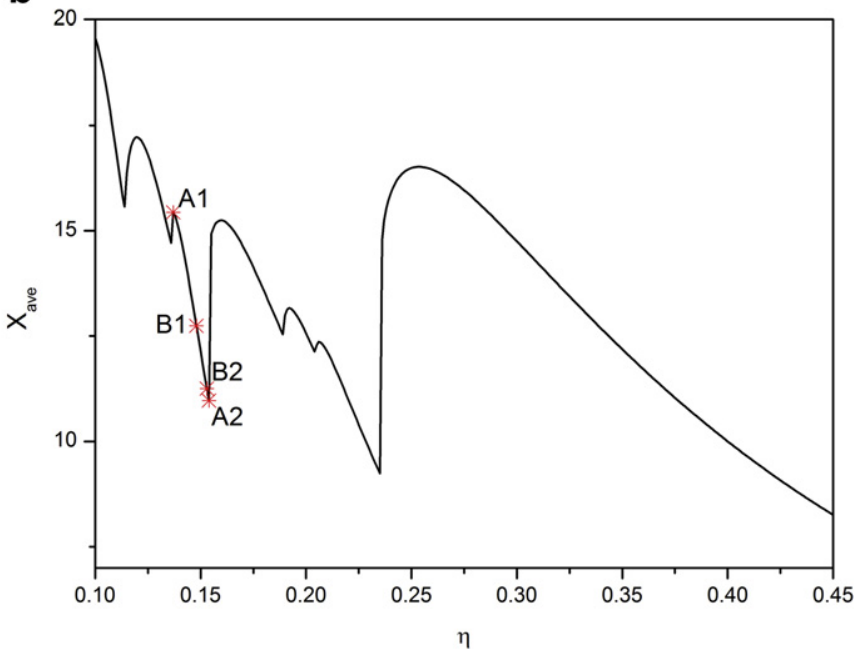

Fig. 5. Frequency-response diagram of $W=0.5$ : (a) complete diagram; (b) local detail diagram of small $\eta$. 


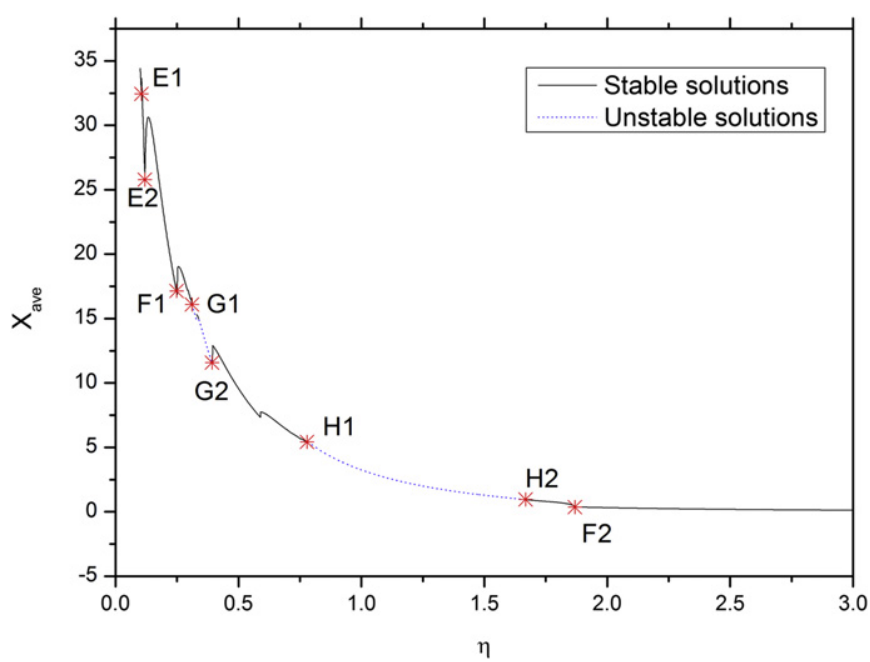

Fig. 6. Frequency-response diagram of $W=0.25$.

Subscript FFT denotes the Jacobian matrix and the residual vector is derived by using FFT in the coefficients of the incremental differential equation.

The elements of Jacobian matrix and residual vector are given by:

$$
\begin{aligned}
& C_{00}=2 \pi k_{0} \\
& A_{0 j}=\pi k_{j}^{C}-\pi j u_{j}^{S}-\pi j^{2} \varphi_{j}^{C} \quad j=1,2, \ldots, N H \\
& B_{0 j}=\pi k_{j}^{S}-\pi j u_{j}^{C}-\pi j^{2} \varphi_{j}^{S} \quad j=1,2, \ldots, N H
\end{aligned}
$$

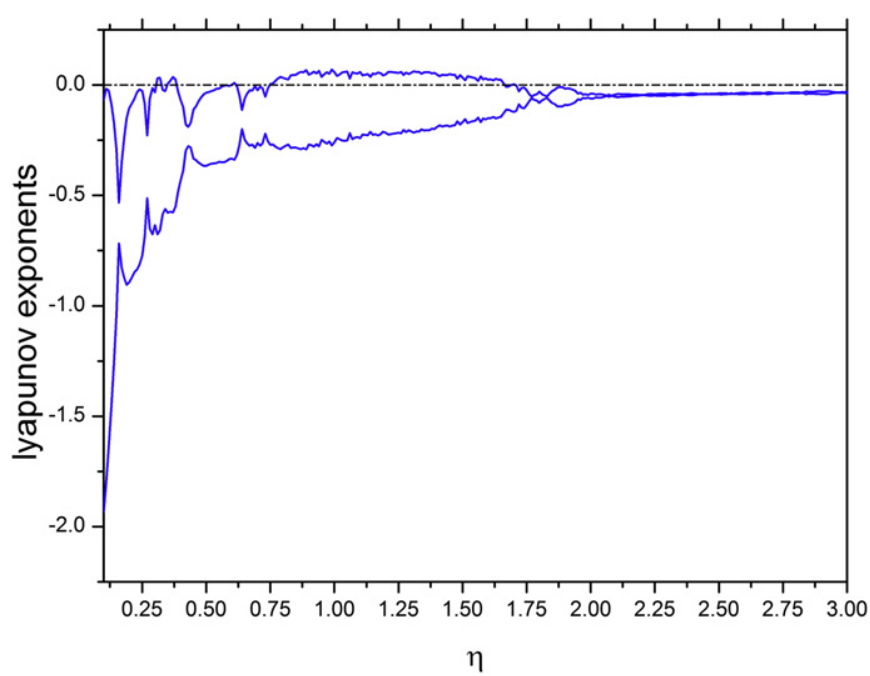

Fig. 7. Lyapunov exponents against $\eta, W=0.25$. 


$$
\begin{aligned}
& C_{i 0}^{C}-\pi k_{i}^{C} \quad i=1,2, \ldots, N H \\
& \begin{array}{l}
A_{i j}^{C}+\frac{\pi}{2}\left\{k_{|i-j|}^{C}+k_{i+j}^{C}-j\left[u_{i+j}^{S}+\operatorname{sgn}(j-i) u_{|i-j|}^{S}\right]-j^{2}\left(\varphi_{i+j}^{C}+\varphi_{|i-j|}^{C}\right)\right\} \quad i \neq j ; i, j=1,2, \ldots, N H \\
A_{i i}^{C}+\frac{\pi}{2}\left[2 k_{0}^{C}+k_{2 i}^{C}-j u_{2 i}^{S}-j^{2}\left(2 \varphi_{0}+\varphi_{2 i}^{C}\right)\right] \quad i=1,2, \ldots N H \\
\quad=1,2, \ldots, N H \\
B_{i j}^{C}=\frac{\pi}{2}\left[k_{i+j}^{S}-\operatorname{sgn}(i-j) k_{|i-j|}^{S}+j\left(u_{i+j}^{C}+u_{|i-j|}^{C}\right)-j^{2}\left(\varphi_{i+j}^{S}-\operatorname{sgn}(i-j) \varphi_{|i-j|}^{S}\right)\right] \quad i \neq j ; i, j \\
B_{i i}^{C}=\frac{\pi}{2}\left[k_{2 i}^{S}+j\left(2 u_{0}+u_{2 i}^{C}\right)-j^{2} \varphi_{2 i}^{S}\right] \quad i=1,2, \ldots N H
\end{array}
\end{aligned}
$$
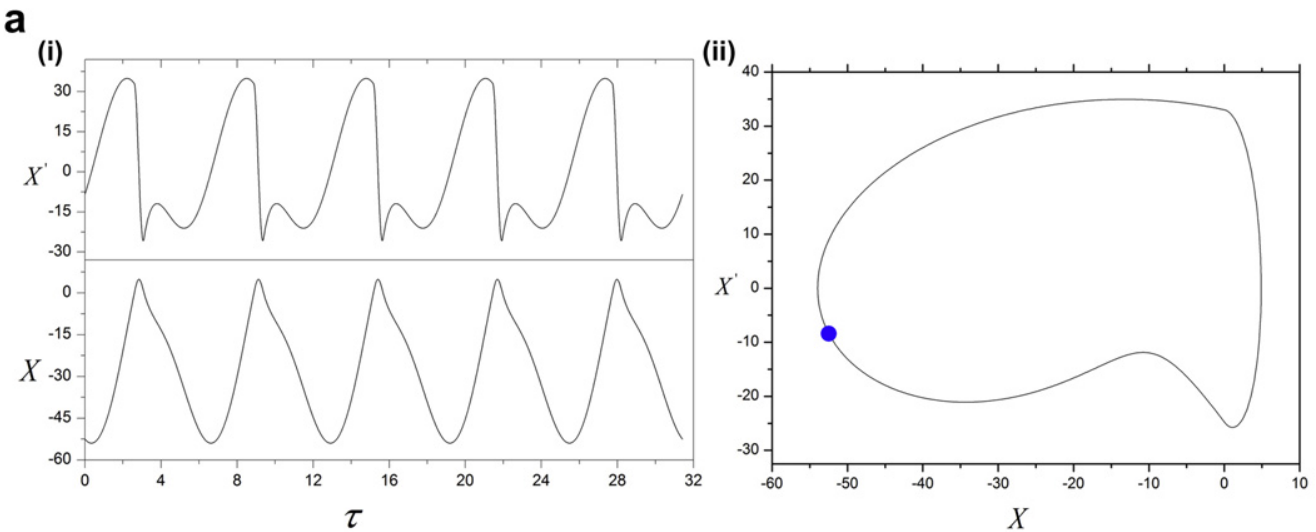

b (i)

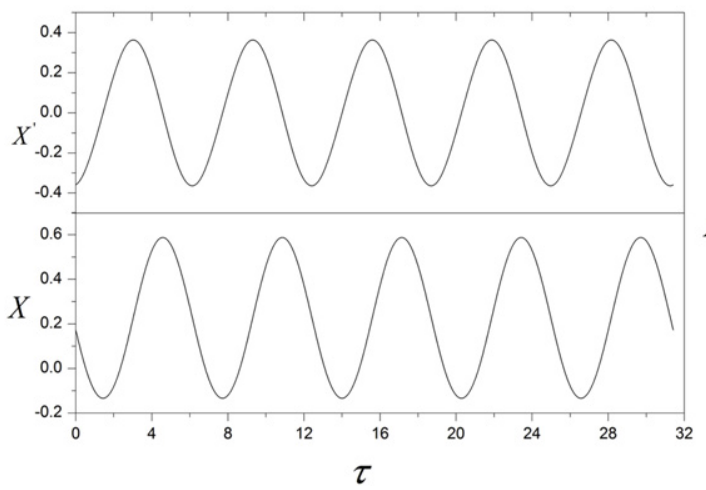

(ii)

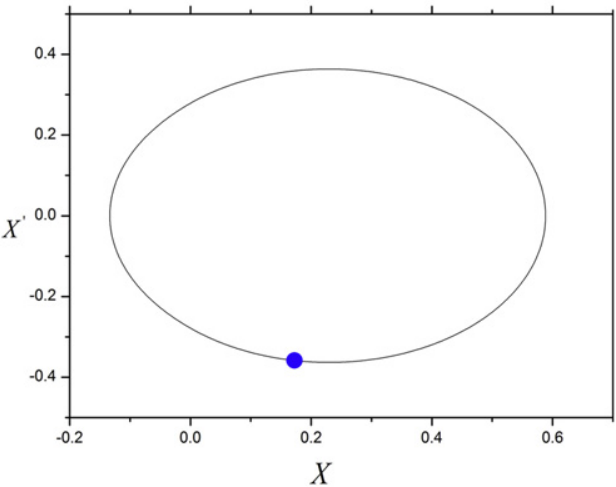

Fig. 8. Typical stable period-1 response- (i) time series, (ii) poincare map and corresponding fixed points, $W=0.25$ : (a). $\eta=0.15$; (b). $\eta=1.9$. 


$$
\begin{aligned}
& C_{i 0}^{S}=\pi k_{i}^{S} i=1,2, \ldots, N H \\
& A_{i j}^{S}=\frac{\pi}{2}\left\{k_{|i+j|}^{S}+\operatorname{sgn}(i-j) k_{|i-j|}^{S}-j\left(u_{|i-j|}^{C}-u_{i+j}^{C}\right)-j^{2}\left(\varphi_{i+j}^{S}+\operatorname{sgn}(i-j) \varphi_{|i-j|}^{S}\right)\right\} \quad i \neq j ; i, j=1,2, \ldots, N H \\
& A_{i i}^{S}=\frac{\pi}{2}\left[k_{2 i}^{S}-2 j u_{0}+j u_{2 i}^{C}-j^{2} \varphi_{2 i}^{S}\right] \quad i=1,2, \ldots N H \\
& B_{i j}^{S}=\frac{\pi}{2}\left\{k_{|i-j|}^{C}-k_{i+j}^{C}+j\left(u_{i+j}^{S}+\operatorname{sgn}(i-j) u_{|i-j|}^{S}\right)-j^{2}\left(\varphi_{|i-j|}^{C}-\varphi_{i+j}^{C}\right)\right\} \quad i \neq j ; i, j=1,2, \ldots, N H \\
& B_{i i}^{S}=\frac{\pi}{2}\left[2 k_{0}-k_{2 i}^{C}+j u_{2 i}^{S}-j^{2}\left(2 \varphi_{0}-\varphi_{2 i}^{C}\right)\right] \quad i=1,2, \ldots N H \\
& R_{0}=2 \pi r_{0}
\end{aligned}
$$

a

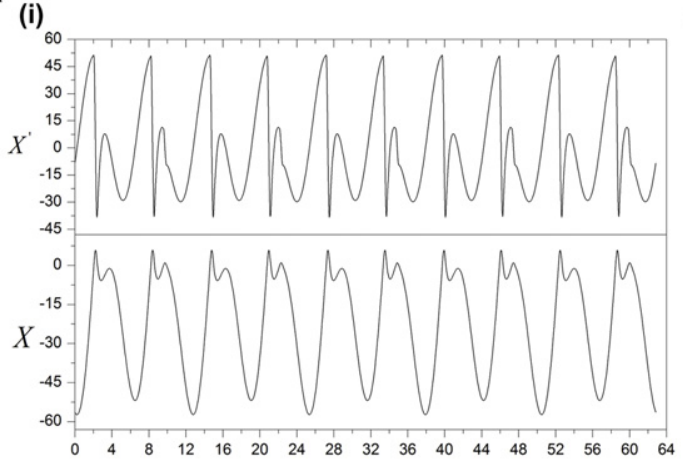

b

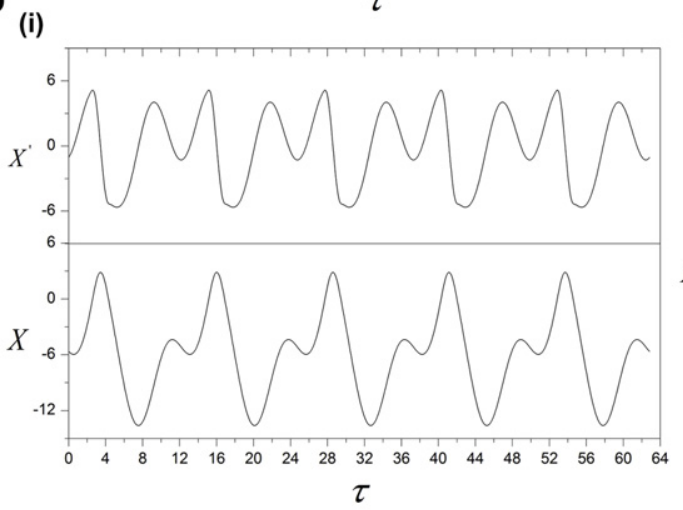

(ii)

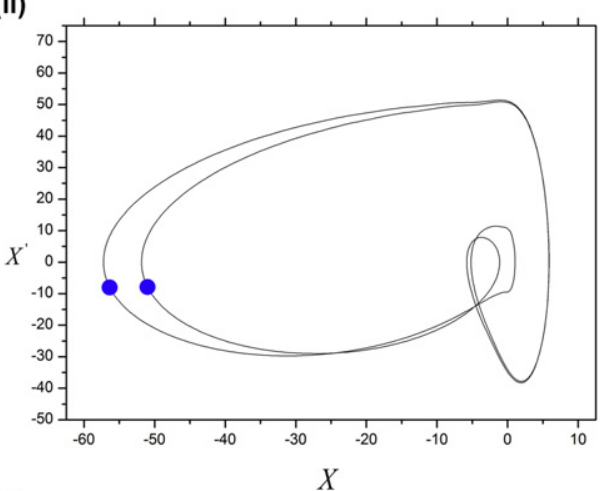

(ii)

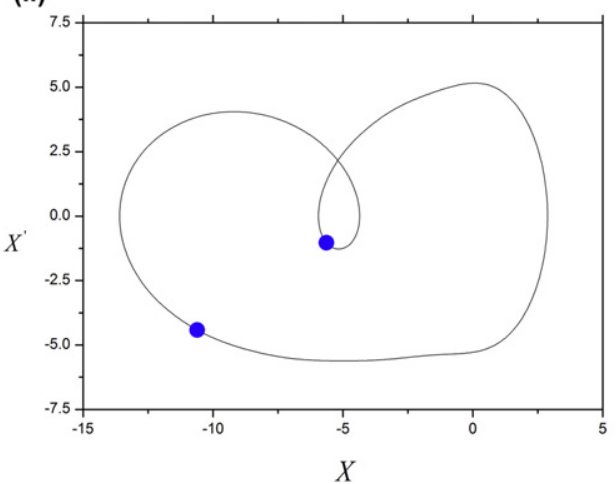

Fig. 9. Typical stable period- 2 response- (i) time series, (ii) poincare map and corresponding fixed points, $W=0.25$ : (a). $\eta=0.11$; (b). $\eta=0.55$. 


$$
\begin{aligned}
& R_{i}^{C}=\pi r_{i}^{C} i=1,2, \ldots, N H \\
& R_{i}^{S}=\pi r_{i}^{S} i=1,2, \ldots, N H
\end{aligned}
$$

The solution at given parameter values can be achieved by an iterative process. An initial value $\mathbf{q}_{0}$ is first given (usually from a corresponding linear solution), then with an FFT the transformed incremental differential equation (16) and corresponding incremental algebraic equation (22) can be derived, from which the increment and the renewed solution will be obtained as:

$$
\begin{aligned}
& {\left[\mathbf{C}_{\mathrm{FFT}}\right]^{i}\{\Delta \mathbf{q}\}^{i}=\left\{\mathbf{R}_{\mathrm{FFT}}\right\}^{i}} \\
& \{\mathbf{q}\}^{i+1}=\{\mathbf{q}\}^{i}+\{\Delta \mathbf{q}\}^{i}
\end{aligned}
$$

This process will be repeated until the maximum of the relative increment is sufficiently small, which means the solution is converged:

$$
\max \left\{\frac{\left|\Delta q_{i}\right|}{\|\mathbf{q}\|}\right\}<\varepsilon i=1,2, \ldots, N H
$$

where $\Delta q_{i}$ is the element of $\Delta \mathbf{q}$ at current step, $\|\mathbf{q}\|$ is the module of $\mathbf{q}, \varepsilon$ is the permissible error.
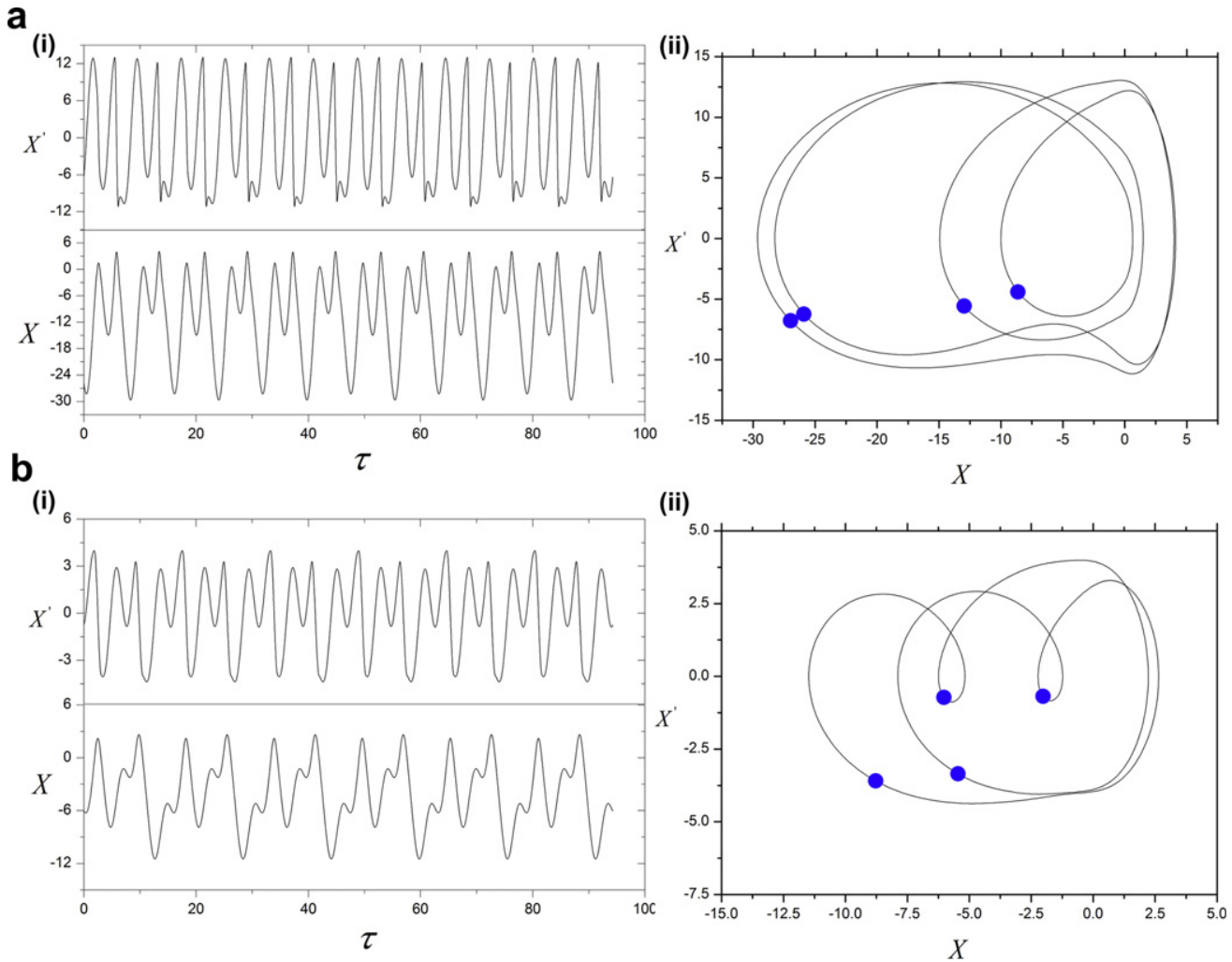

Fig. 10. Typical stable period- 4 response- (i) time series, (ii) poincare map and corresponding fixed points, $W=0.25$ : (a). $\eta=0.3$; (b). $\eta=0.65$. 
$\mathbf{a}_{(\mathrm{i})}$

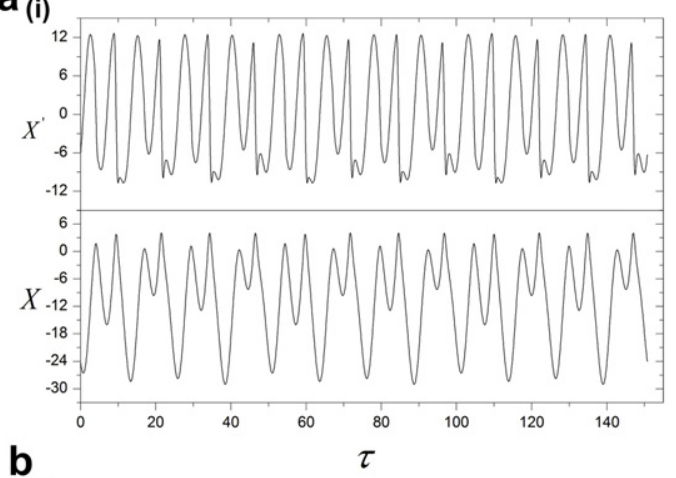

(i)

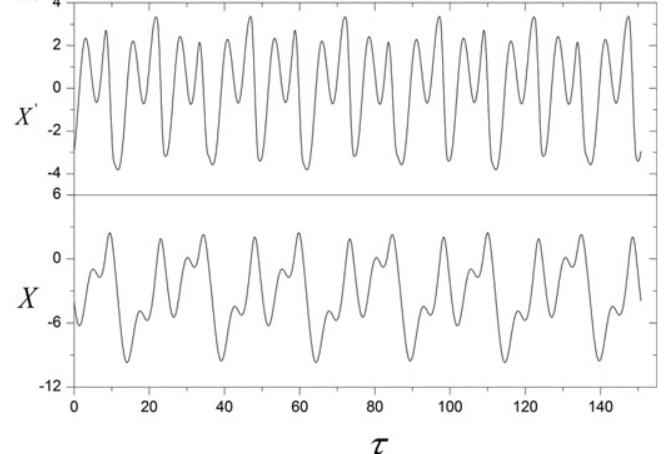

(ii)

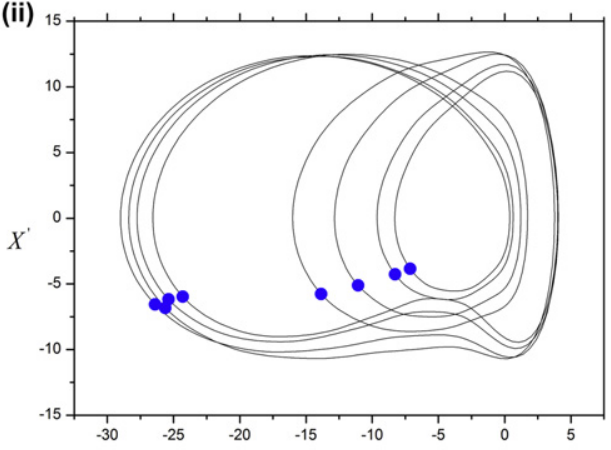

(ii)

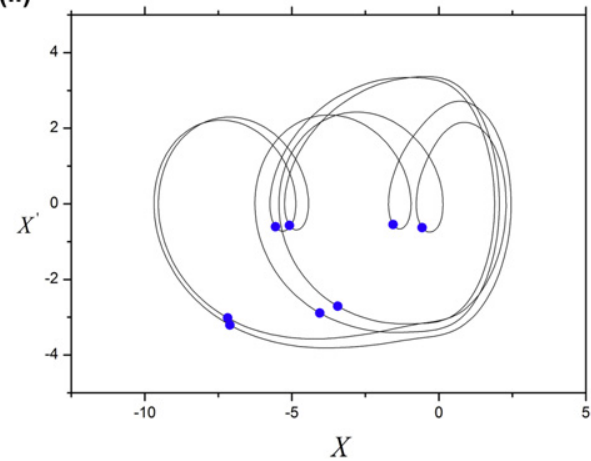

Fig. 11. Typical stable period-8 response- (i) time series, (ii) poincare map and corresponding fixed points, $W=0.25$ : (a). $\eta=0.307$; (b). $\eta=0.72$.

A flow chart of the present solving procedure of IHB method with FFT is given in Fig. 2 . As the previous deriving course in this section and Fig. 2 shows, intrinsically the basic idea of IHB method is to expand a differential equation and transform it to nonlinear algebraic equations with coefficients of Fourier series as the unknown variables by harmonic balance (Galerkin average procedure). Since solving the nonlinear algebraic equations directly is generally difficult, so that IHB method introduces an incremental step, and solve the nonlinear equations by Newton-Raphson iteration.
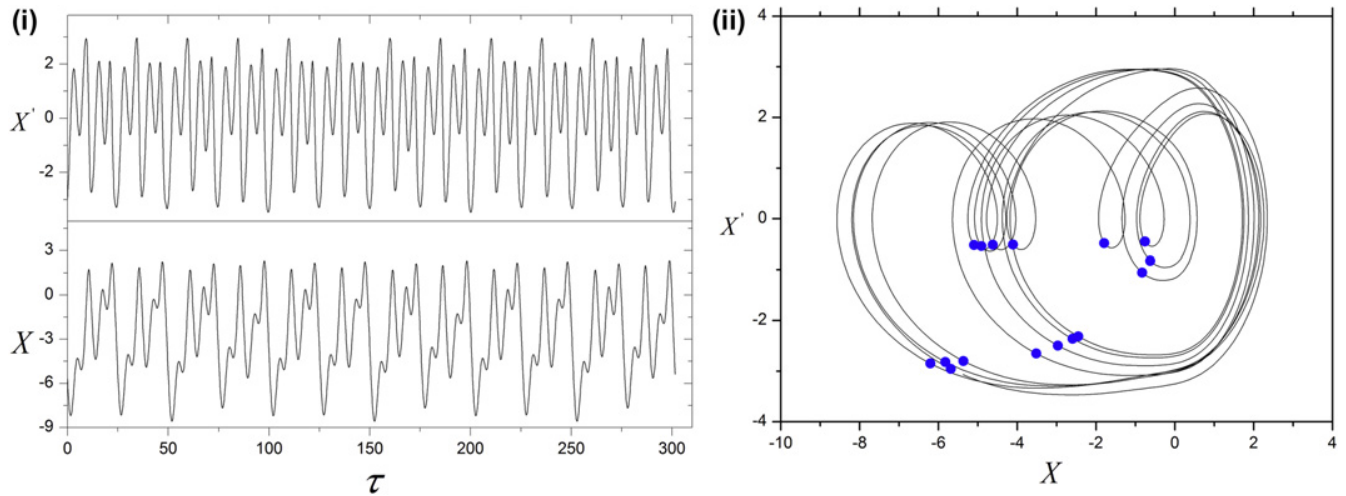

Fig. 12. Typical stable period-16 response- (i) time series, (ii) poincare map and corresponding fixed points, $W=0.25, \eta=0.775$. 
As long as the solution at a given parameter is obtained, the solution at a new nearby system parameter value can be easily obtained with this solution as the new initial value, which means that the parameter analysis can be easily accomplished. Both stable and unstable solutions, and both fundamental and subharmonic resonant solutions can be directly obtained from IHB method. The stability of the periodic solutions can be investigated by Floquet theory. The transition matrix of the perturbed governing equation can be evaluated by the method summarized in Friedmann's paper [23]. To get the $L$-th order subharmonic solutions, we assume the solution and its increment of the form of:

$$
\begin{aligned}
& x(t)=a_{0}+\sum_{i=1}^{N H}\left(a_{i} \cos \frac{i t}{L}+b_{i} \sin \frac{i t}{L}\right) \\
& \Delta x(t)=a_{0}+\sum_{i=1}^{N H}\left(\Delta a_{i} \cos \frac{i t}{L}+\Delta b_{i} \sin \frac{i t}{L}\right)
\end{aligned}
$$

\section{Results and discussions}

\subsection{Accuracy of IHB method with FFT and comparison with numerical integration results}

Table 1 gives the maximum values of the residual term $R$ in a period. It indicates that generally the maximum residual value can be reduced by adding more harmonic terms in the assumed solution
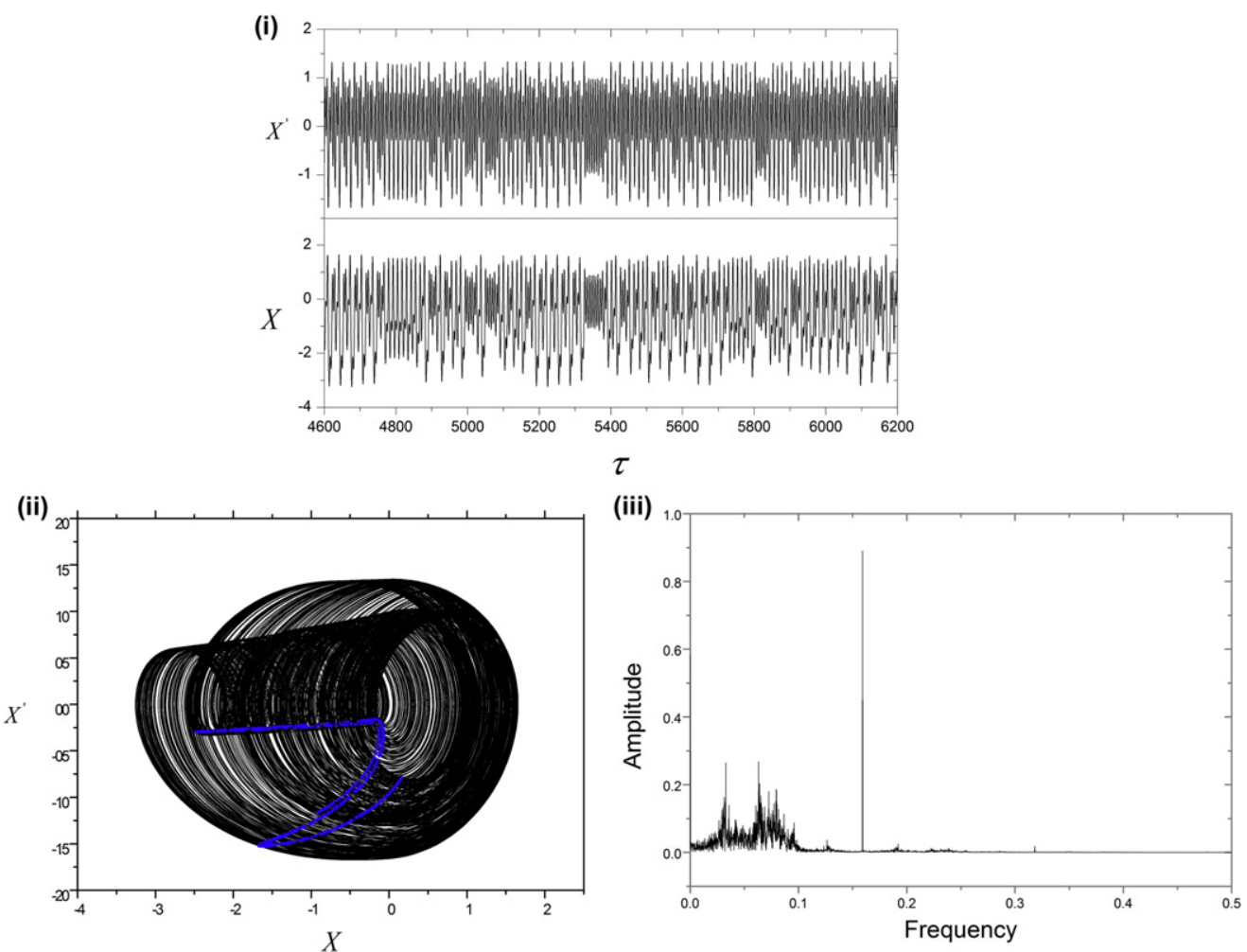

Fig. 13. Chaotic response at $\eta=1.2$ (simulation time $=2000 \pi$ ): (i) time series; (ii) poincare map and corresponding fixed points; (iii) fourier spectrum. 
series. While for period- 1 solution, merely 5 harmonic terms can achieve a desirable accuracy. Fig. 3(a)-(d) compare the phase planes of the period-1 solutions obtained by IHB method with the fourth order Runge-Kutta method, and the results from the two approaches coincide well.

\subsection{Effect of dimensionless net buoyancy $\mathrm{W}$, bifurcations and a path to chaotic motions}

The frequency-response curves are traced by IHB method with iterative procedure described in Section 3 at small damping ratio $(\zeta=0.1)$. The frequency-response curves under different dimensionless net buoyancy $W$ are summarized in Fig. 4. The figure shows plots of the average response amplitude $X_{\text {ave }}\left(=\left(X_{\max }-X_{\min }\right) / 2\right)$ against the frequency ratio $\eta$ (varying from 0.1 to 3 ) and the dimensionless net buoyancy $W$ ( varying from 0.25 to 5 ). It can be seen that the peak value of average response $X_{a v e}$ increases dramatically with the decrease of dimensionless net buoyancy, and the response amplitude at small frequency ratio $\eta$ becomes large. It is worth noticing that when $W>5$ the
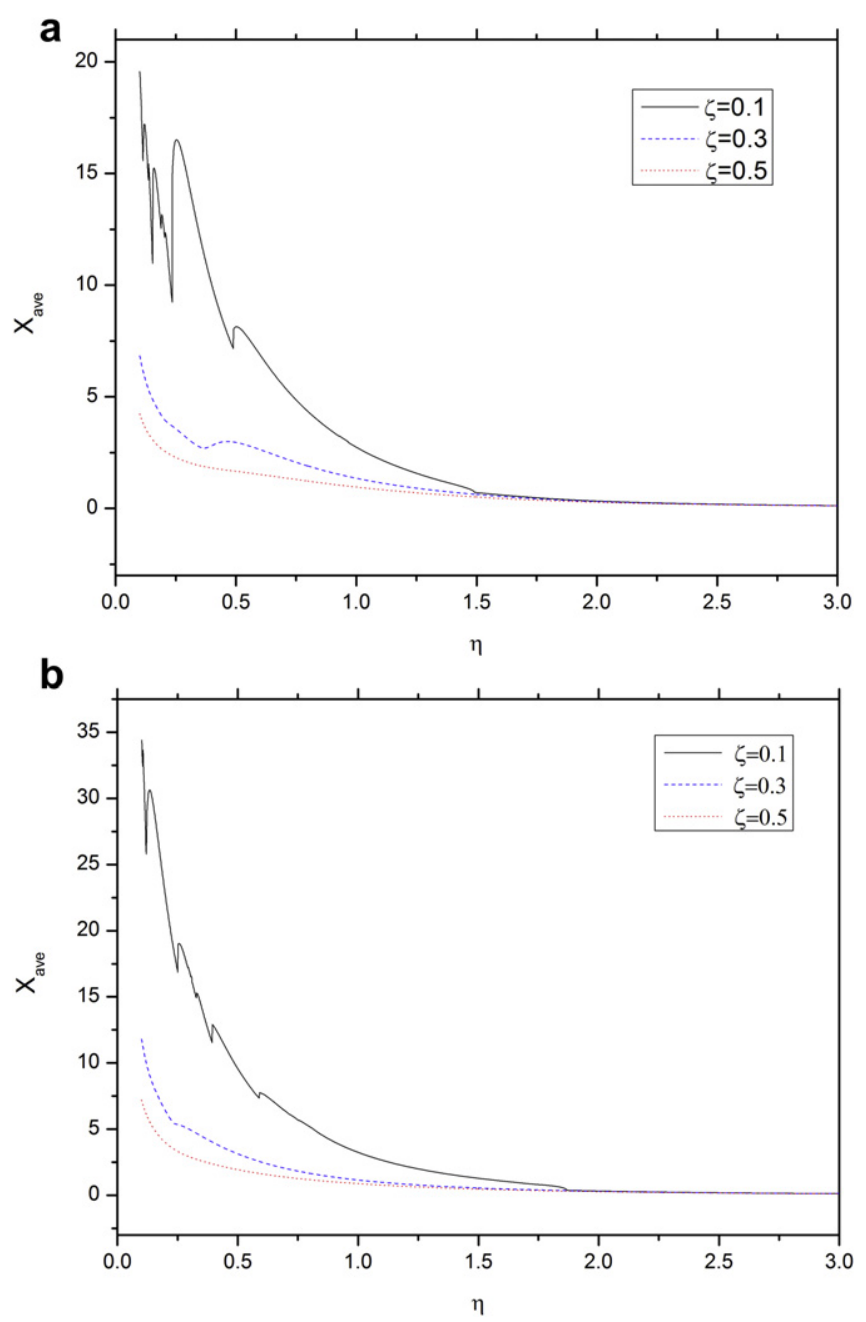

Fig. 14. Response diagram: (a) $W=0.5$; (b) $W=0.25$. 
(i)

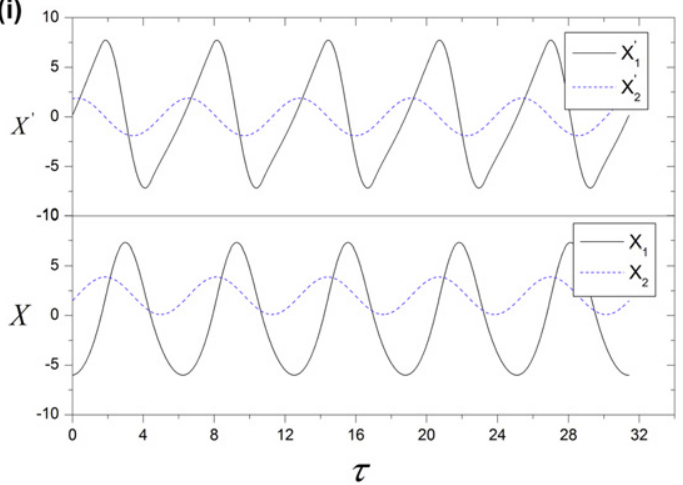

(ii)

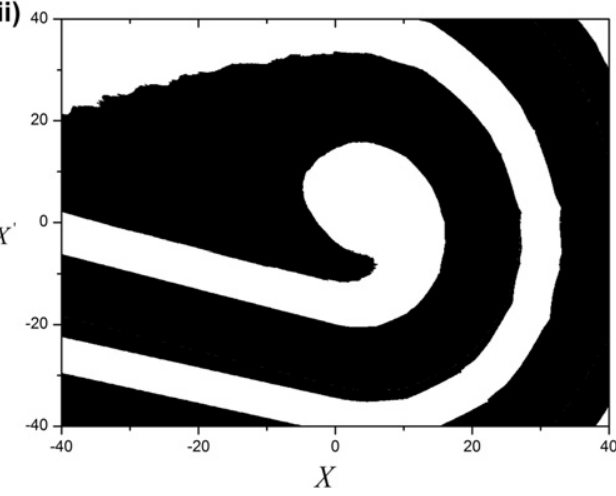

Fig. 15. Two coexistent period- 1 solution, $\zeta=0.1, W=2.0, \eta=0.7$ : (i) time series, (ii) domains of attraction ( $400 \times 400$ cells).

minimum dimensionless displacement $X_{\min }$ remains positive, which indicates that the nonlinear system (Eq. (3)) will degenerate to a classic linear system. The main resonance peak moves towards the left side of $\eta=1$ as $W$ decreases from 5 . When $W \geq 1$, Period- 1 response is stable.

Fig. 5(a), (b) gives the frequency-response curves when $W=0.5$, in which Fig. 4 (b) is the local detail of the left side in Fig. 4 (a) $(\eta=0.1-0.45)$. Taking frequency ratio $\eta$ as bifurcation parameter, Period-1 solution becomes unstable at the marked points of $\mathrm{A} 1(\eta=0.137)$ and $\mathrm{A} 2(\eta=0.154), \mathrm{C} 1(\eta=0.49)$ and $\mathrm{C} 2(\eta=1.489)$ in Fig. 5 (a). Between A1-A2 and C1-C2, the Floquet multiplier of the transition matrix moves out of the unit circle along the -1 direction, which means at these points period- 1 solutions bifurcate to period-2 solution through period doubling bifurcation. Similarly the period-2 solution further becomes unstable at B1 $(\eta=0.148), \mathrm{B} 2(\eta=0.153)$ and D1 $(\eta=0.929)$, D2 $(\eta=0.966)$; and bifurcates to period-4 solution between B1 and B2, D1 and D2.

As the dimensionless net buoyancy $W$ decreases to 0.25, it is shown in Fig. 6 that between E1 $(\eta=0.105)$ and E2 $(\eta=0.12), \mathrm{F} 1(\eta=0.248)$ and F2 $(\eta=1.869)$ the period -1 solutions become unstable and bifurcate to period-2 solutions. Eventually, between G1 $(\eta=0.311)$ and G2 $(\eta=0.393), \mathrm{H} 1$ $(\eta=0.779)$ and $\mathrm{H} 2(\zeta=0.1)$, the system will go to chaotic through a series of period doubling bifurcations. The Lyapunov exponents are calculated by Wolf's method [25] as shown in Fig. 7. The figure shows that between $\mathrm{G} 1$ and G2, $\mathrm{H} 1$ and $\mathrm{H} 2$, the maximum Lyapunov exponents are positive, which confirms the conclusion that the system goes to chaotic. Typical stable response of priod-1 and bifurcated solutions obtained by IHB method with FFT are given in Figs. 8-12. The chaotic responses are
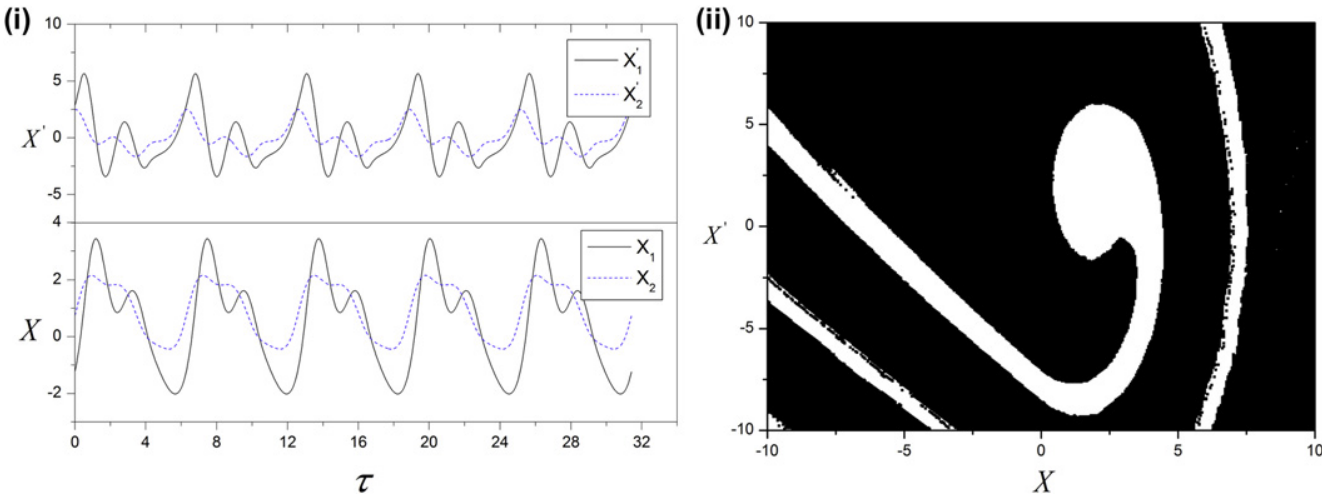

Fig. 16. Two coexistent period- 1 solution, $\zeta=0.1, W=1.0, \eta=0.364$ : (i) time series, (ii) domains of attraction $(400 \times 400$ cells). 
obtained by the fourth order Runge-Kutta method. Fig. 13 (a)-(c) show the chaotic motion time series, Poincare map with corresponding fixed points and the Fourier spectrum respectively at $\eta=1.2$.

\subsection{Effect of damping ratio $\zeta$, existence of multiple solutions}

The frequency-response curves are also traced for different damping ratio $\zeta$. Fig. 14 (a) and (b) compare the response curves of different damping ratio. The diagrams show that the response amplitudes decrease greatly with the increasing of $\zeta$. Besides, stability analysis indicates that when $\zeta \geq 0.3$, period-1 solution is always stable despite of $\eta$ and $W$. Multiple solutions are observed at small damping ratio $(\zeta=0.1)$. Two coexistent stable period- 1 solutions obtained by IHB method with different initial values are shown in Fig. 15 (a) and Fig. 16 (a) respectively. The domains of attraction of corresponding solutions are analyzed by Interpolated Cell Mapping (ICM) technique [24]. The diagrams of domains of attraction are shown in Fig. 15 (b) and Fig. 16 (b), in which the black dots correspond to the initial conditions ending in the period one attractor with larger amplitude that plotted in solid lines in Fig. 15 (a) and (b).

\section{Concluding remarks}

In this paper, the nonlinear dynamics of a submerged floating moored structure subjected to vertical excitation with possible slackness in the mooing system are studied by incremental harmonic balance method with FFT. By deriving the dimensionless governing equation, it is found that only three independent parameters, frequency ratio $\eta$, damping ratio $\zeta$ and dimensionless net buoyancy $W$, exists. Due to the fact that the restoring force term is a function of the unknown displacement and could barely be expressed in an explicit form of time, IHB method with an implement of FFT is used before the Galerkin average procedure, which is proved to be of high efficiency and accuracy. The results show that the dimensionless net buoyancy $W$, that is the ratio of net buoyancy to the excitation amplitude, affects the system dynamic characteristics greatly. The response amplitude increases dramatically as $W$ decreases; when $W$ decreased as small as 0.25 , the system will go to chaotic through a series of period doubling bifurcations. The system nonlinearity becomes stronger as damping ratio $\zeta$ decreases; multiple solutions are observed when $\zeta=0.1$. These results provide a profound understanding for the nonlinear course of this type of structures, and can thus serve as some important references for the structure design consideration.

\section{Acknowledgment}

This paper is supported by National Natural Science Foundation of China (nos. 10532070, 10772178) and Knowledge Innovation Program of Chinese Academy of Sciences (no. KJCX2-YW-L07).

\section{References}

[1] Liu Y, Yu-cheng L, Bin T, Sheng D. Wave motion over a submerged breakwater with an upper horizontal porous plate and a lower horizontal solid plate. Ocean Engineering 2008;35(16):1588-96.

[2] Rambabu AC, Mani JS. Numerical prediction of performance of submerged breakwaters. Ocean Engineering 2005;32(10): 1235-46.

[3] Sundar V, Sundaravadivelu R, Kalyani M. Forces due to oblique waves on a submerged open moored cylinder in deep waters. Ocean Engineering 2005;32:651-66.

[4] Ahren D. Submerged floating tunnels-a concept whose time has arrived. Tunnelling and Underground Space Technology 1997;12(2):317-36.

[5] Youshi H, Fei G. Dynamic response and structural integrity of submerged floating tunnel due to hydrodynamic load and accidental load. Procedia Engineering 2010;4:35-50.

[6] Liu FC. Snap loads in lifting and mooring cable systems induced by surface wave conditions. Port Hueneme, CA: Technical Note N-1288, Naval Civil Engineering Laboratory; 1973.

[7] Niedzwecki JM, Thampi SK. Snap loading of marine cable systems. Applied Ocean Research 1991;13(1):2-11.

[8] Huang S, Vassalos D. A numerical method for predicting snap loading of marine cables. Applied Ocean Research 1993; 15(4):235-42.

[9] Patel MH, Park HI. Dynamics of tension leg platform tethers at low tension. Part I-mathieu stability at large parameters. Marine Structures 1991;4(3):257-73.

[10] Patel MH, Park HI. Tensioned buoyant platform tether response to short duration tension loss. Marine Structures 1995; 8(5):543-53. 
[11] Vassalos D, Huang S. Dynamics of small-sagged taut-slack marine cables. Computers \& Structures 1996;58(3):557-62.

[12] Plaut RH, Archilla JC, Mays TW. Snap loads in mooring lines during large three-dimensional motions of a cylinder. Nonlinear Dynamics 2000;23(3):271-84.

[13] Wei L, Fei G, Lei W, Youshi H. On the slack phenomena and snap force in tethers of submerged floating tunnels under wave conditions. Marine Structures 2011;24(4):358-76.

[14] Vassalos D, Huang S, and Kourouklis A. Experimental investigation of snap loading of marine cables. In: proceedings of the fourteenth international offshore and polar engineering conference; 2004.

[15] Kunisu H, Mizuno S, Mizuno Y, Saeki H. Study on subemerged floating tunnel characteristics under the wave condtion. In: proceedings of the fourth international offshore and polar engineering conference; 1994.

[16] Cheung YK, Lau SL. Incremental time-space finite strip method for non-linear structural vibrations. Earthquake Engineering \& Structural Dynamics 1982;10(2):239-53.

[17] Lau SL, Cheung YK. Amplitude incremental variational principle for non-linear vibration of elastic-systems. Journal of Applied Mechanics 1981;48(4):959-64.

[18] Cheung YK, Chen SH, Lau SL. Application of the incremental harmonic-balance method to cubic nonlinearity systems. Journal of Sound and Vibration 1990;140(2):273-86.

[19] Lau SL, Zhang WS. Nonlinear vibrations of piecewise-linear systems by incremental harmonic-balance method. Journal of Applied Mechanics 1992;59(1):153-60.

[20] Raghothama A, Narayanan S. Bifurcation and chaos of an articulated loading platform with piecewise non-linear stiffness using the incremental harmonic balance method. Ocean Engineering 2000;27(10):1087-107.

[21] Xu L, Lu MW, Cao Q. Bifurcation and chaos of a harmonically excited oscillator with both stiffness and viscous damping piecewise linearities by incremental harmonic balance method. Journal of Sound and Vibration 2003;264(4):873-82.

[22] Leung AYT, Chui SK. Nonlinear vibration of coupled duffing oscillators by an improved incremental harmonic-balance method. Journal of Sound and Vibration 1995;181(4):619-33.

[23] Friedmann P, Hammond CE, Woo TH. Efficient numerical treatment of periodic systems with application to stability problems. International Journal for Numerical Methods in Engineering 1977;11(7):1117-36.

[24] Tongue BH, Gu K. Interpolated cell mapping of dynamical-systems. Journal of Applied Mechanics 1988;55(2):461-6.

[25] Wolf A, Jack BS, Harry LS, John AV. Determining lyapunov exponents from a time-series. Physica D 1985;16(3):285-317. 